\title{
Caspase-8 cleaves its substrates from the plasma membrane upon CD95-induced apoptosis
}

\author{
J Beaudouin ${ }^{1}$, C Liesche ${ }^{1}$, S Aschenbrenner ${ }^{1}$, M Hörner ${ }^{1,2}$ and R Eils ${ }^{\star, 1}$
}

Apoptosis occurs through a tightly regulated cascade of caspase activation. In the context of extrinsic apoptosis, caspase-8 is activated by dimerization inside a death receptor complex, cleaved by auto-proteolysis and subsequently released into the cytosol. This fully processed form of caspase- 8 is thought to cleave its substrates BID and caspase-3. To test if the release is required for substrate cleavage, we developed a novel approach based on localization probes to quantitatively characterize the spatial-temporal activity of caspases in living single cells. Our study reveals that caspase-8 is significantly more active at the plasma membrane than within the cytosol upon CD95 activation. This differential activity is controlled by the cleavage of caspase-8 prodomain. As a consequence, targeting of caspase-8 substrates to the plasma membrane can significantly accelerate cell death. Subcellular compartmentalization of caspase-8 activity may serve to restrict enzymatic activity before mitochondrial pathway activation and offers new possibilities to interfere with apoptotic sensitivity of the cells.

Cell Death and Differentiation (2013) 20, 599-610; doi:10.1038/cdd.2012.156; published online 11 January 2013

Apoptosis is coordinated by the activity of initiator and effector caspases. ${ }^{1-4}$ While effector caspases are dimeric zymogens that become activated by cleavage, initiator caspases are normally expressed as monomeric zymogens and their activity is initiated by dimerization in a multimeric complex. ${ }^{5,6}$ The initiator caspase procaspase-8 dimerizes in the death-inducing signaling complex (DISC) formed around activated death receptors. ${ }^{7}$ In the context of CD95, the DISC contains clustered receptors bound to the adaptor protein FADD. FADD can recruit several proteins, ${ }^{8,9}$ including procaspase-8 and -10 through their prodomain. In type I cells, active caspase-8/10 directly cleaves and activates effector caspase-3/-7, thus inducing apoptosis. In type II cells, this activation is blocked by XIAP, but cleavage of BID by active caspase-8/10 induces mitochondrial outer membrane permeabilization (MOMP), followed by initiator caspase- 9 activation and release of XIAP-inhibitor SMAC, leading to massive caspase-3/7 activity. ${ }^{10,11}$

Biochemical and structural studies showed that dimerization but also cleavage in the catalytic subunit of caspase- 8 is required for efficient cleavage of caspase-3 and BID and for apoptosis. ${ }^{12-14}$ Supporting this, the non-cleavable D387A caspase-8 mutant compromises apoptosis in mice. ${ }^{15}$ Although caspase-8 dimerization generates some activity, cleavage in the catalytic units likely stabilizes the active form and increases activity. ${ }^{13,16}$

Caspase- 8 is also cleaved between the prodomain and the catalytic unit of the enzyme, at D210 and D216, ${ }^{17,18}$ and subsequently released from the DISC. Active caspase-8 can be detected on the plasma membrane, before release, with fluorescent inhibitors. ${ }^{19}$ In contrast, by designing a procaspase-8 artificially dimerized on the plasma membrane, Martin et al. ${ }^{18}$ suggested that the cytosolic release is necessary to cleave caspase-3 and BID. Other studies proposed that fully processed caspase- 8 translocates to the surface of mitochondria to cleave BID. ${ }^{20,21}$ However, released caspase- 8 is less active than inside the DISC in vitro, ${ }^{12}$ rather suggesting that cytosolic caspase-3 and BID encounter active caspase-8 at the DISC and are cleaved there. Still, the standard model is that the fully processed cytosolic form of caspase- 8 cleaves caspase-3 and BID. ${ }^{22,23}$

To resolve these partially contradicting findings, we developed a novel assay that allows to quantitatively and spatially characterize caspase-8 activity in single living cells. We designed probes by fusing fluorescent proteins to localization domains through cleavage sites. Caspase activity is measured by monitoring the fluorescent protein redistribution after cleavage. Here, we show that upon CD95 activation, caspase- 8 is mostly active at the plasma membrane and less active inside the cytoplasm. As a consequence, targeting apoptotic proteins to the plasma membrane strongly promotes the apoptotic response. Our results show that in the process of apoptosis, caspase substrate cleavage is in part regulated by the spatially defined enzyme and substrate concentrations.

\footnotetext{
${ }^{1}$ Department for Bioinformatics and Functional Genomics, Division of Theoretical Bioinformatics, German Cancer Research Center (DKFZ), Institute for Pharmacy and Molecular Biotechnology (IPMB) and BioQuant, Heidelberg, Germany

${ }^{*}$ Corresponding author: R Eils, Department for Bioinformatics and Functional Genomics, German Cancer Research Center (DKFZ), Institute for Pharmacy and Molecular Biotechnology (IPMB) and BioQuant Im Neuenheimer Feld 267, Heidelberg, 69120, Germany. Tel: +49 6221 5451290; Fax: +49 6221 5451488; E-mail: r.eils@dkfz-heidelberg.de

${ }^{2}$ Present address: Faculty of Biology and Center for Biological Signalling Studies, 79104 Freiburg, Germany.

Keywords: apoptosis; live-cell imaging; quantitative biology; caspase sensor

Abbreviations: DISC, death-inducing signaling complex; BID, BH3-interacting death agonist; MOMP, mitochondrial outer membrane permeabilization; NES, nuclear export signal; Myr-Palm, myristoylation-palmitoylation; ER, endoplasmic reticulum; LZ-sCD95L, leucine zipper-soluble CD95 ligand; mGFP, monomeric green fluorescent protein; YFP, yellow fluorescent protein; EBFP2, enhanced blue fluorescent protein 2; XIAP, X-linked inhibitor of apoptosis protein; FRET, fluorescence resonance energy transfer; DMEM, Dulbecco's modified eagle medium; LSCM, laser scanning confocal microscope

Received 15.3.12; revised 27.9.12; accepted 4.10.12; Edited by G Salvesen; published online 11.1.13
} 


\section{Results}

Development of localization probes to quantitatively characterize the spatio-temporal activity of caspases in living cells. To spatially resolve caspase activity and to compare different substrate specificities within single cells, we developed genetically encoded fluorescent probes targeted to different functionally relevant subcellular compartments. The probes were composed of localization domains fused to fluorescent proteins through cleavable linkers (Figure 1a). The fluorescent protein, initially trapped by the localization domain, is released upon cleavage, a process that can be visualized and quantified.

Cytokeratin-8, calnexin ${ }^{24}$ and MitoNEET ${ }^{25}$ were used to target probes to the cytoskeleton, endoplasmic reticulum (ER) and the outer membrane of the mitochondria, respectively, to measure enzymatic activity inside the cytoplasm with no contribution of plasma membrane activity. Nuclear export signal (NES) was used to detect activity in the cytosolic space including the plasma membrane. A myristoylationpalmitoylation (Myr-Palm) domain combined with a spacer made of snap tags (Supplementary Figure 1a) targeted probes to the plasma membrane, which can be cleaved by plasma membrane and soluble cytosolic enzymes. Finally, activity in the nucleus was measured by targeting probes to histones using $\mathrm{H} 2 \mathrm{~B}$ (Figure 1b).

Upon cleavage, confirmed by western blot (Supplementary Figure 1b), fluorescent proteins equilibrate by diffusion between the cytosol and the nucleus, on a short and neglectable time scale of $\sim 2 \min ^{26,27}$ We monitored this process and compared different probes within the same cell by combining mCherry with mGFP, EYFP or EBFP2 probes using time-lapse confocal microscopy. Exchanging colors gave similar behaviors (Supplementary Figure 2a) and were photostable during the apoptotic process (Supplementary Figures 2b and c).
Nuclear and cytosolic intensities of cleaved cytoplasmic and nuclear probes, respectively, were measured over time and normalized to allow comparison between probes within one cell and between different cells (see Supplementary Material for the workflow). To statistically analyze the data independently of cell-to-cell variation of time of death, we normalized time to 1 at the formation of apoptotic body for each cell. All control non-cleavable probes remained intact until apoptotic body formation, except the NES probe that started equilibrating 2 min before (Supplementary Figure $2 d$ ). Taken together, these localization domain probes allow a precise measurement of caspase activity in a spatio-temporally resolved manner in living cells.

The NES probe derived from the BID cleavage sequence ELQTDG is mostly cleaved by caspase-8 before apoptotic body formation. In order to characterize the temporal pattern of caspase-8 activity, we first systematically compared the sequence cleavage of different caspase-8 substrates using the NES probe. These sequences were GIETDS for caspase-3, PPVETDS for caspase-8, LLEVDG for cFLIP-L and ELQTDG for BID. To amplify caspase-8 activity and facilitate its visualization, we first coexpressed EYFP and mCherry probes in HeLa cells stably overexpressing CD95 (HeLa-CD95). mCherry was always tagged to ELQTDG as reference. Apoptotic bodies typically formed 40-50 min after addition of LZ-sCD95L. During that period, starting at $10 \mathrm{~min}$, GIETDS, PVETDS, LLEVDG and ELQTDG probes were gradually and similarly cleaved (Figures 2a-e). We therefore focused on one cleavage sequence for the rest of the study, namely ELQTDG for BID.

To determine the contribution of caspase-8 and downstream caspases on ELQTDG cleavage, we measured effector caspase activity using an NES probe containing DEVDR, which can be cleaved by caspase-3, less efficiently
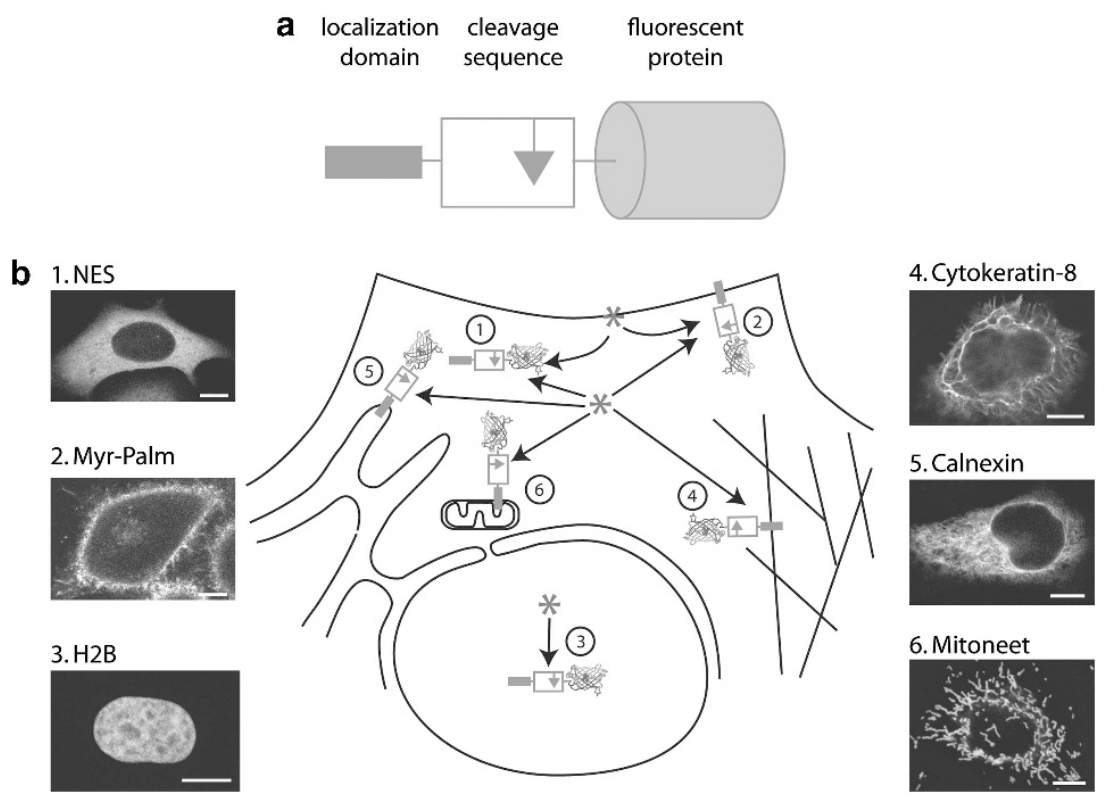

Figure 1 (a) Schematic representation of localization probes. (b) Localization of probes before cleavage: cytosol (1), plasma membrane (2), nucleus (3), cytoskeleton (4), endoplasmic reticulum (5) and mitochondria (6). Arrows indicate possibilities of cleavage depending on the localization of the enzyme (denoted by red star). A full color version of this figure is available at Cell Death and Differentiation journal online. 
by caspase-7 and very weakly by caspase-8. ${ }^{28,29}$ As already observed in type II cells, ${ }^{28}$ caspase- 3 activity was significantly delayed compared with caspase-8, appearing shortly before cell death (Figures $2 f$ and $g$ ). Caspase-3 is therefore not expected to cleave ELQTDG probes in the early phase of caspase-8 activity. To further exclude NES-ELQTDG probe cleavage by downstream caspases, we inhibited caspase-3/7/ 9 activity by overexpressing mCherry-XIAP. This overexpression blocked apoptosis (Figure $2 \mathrm{~h}$ ) and strongly reduced NESDEVDR-EYFP cleavage (Figure 2i). In contrast, NESELQTDG-EBFP2 was cleaved at the same rate as in control cells in the time interval where those cells were alive (Figure 2j). This shows that, at least until apoptotic body formation, NESELQTDG cleavage essentially represents caspase-8 activity.

Caspase-8 probes are most efficiently cleaved at the plasma membrane upon CD95 activation. Full processing of caspase-8, including release from the DISC, is generally thought to be necessary to cleave caspase- 3 and BID. ${ }^{18}$ In this case, the different localization probes would all be cleaved at the same rate. In contrast, if caspase- 8 can cleave its substrates from the plasma membrane, already after cleavage in the catalytic domain, we expect NES and MyrPalm probes to be cleaved more efficiently than probes that cannot access the plasma membrane. To discriminate between these hypotheses, we compared the cleavage of ELQTDG probes fused to EYFP or mGFP and targeted to different intracellular compartments, using NES-ELQTDGmCherry as a reference (Figure 3). Strikingly, we observed that cytoplasmic probes that cannot access the plasma membrane, targeted to cytokeratin filaments and to the ER and mitochondria surfaces, were equally inefficiently cleaved compared with NES-ELQTDG-mCherry (Figures 3a-d). In contrast, the caspase-3-specific cleavage site DEVDR showed similar cleavage efficiency for all probes (Supplementary Figure 2e), excluding a hindrance of the cleavage linker. In HeLa cells overexpressing mCherryXIAP, we observed that the ER probe was cleaved at a lower rate than the NES probe and with a delay roughly coinciding with the time of apoptotic body formation of control cells (Figure 3e). However, as NES-DEVDR was also partially cleaved during this late phase (Figure 2i), we cannot exclude the involvement of downstream caspases during this period.

The cleavage of the nuclear probe showed that caspase-8 nuclear activity occurs only after the formation of apoptotic bodies (Figure 3f). In contrast, we observed a strong activity at the plasma membrane, leading to a complete Myr-Palmsnap3-ELQTDG probe cleavage much before apoptotic body formation (Figure $3 \mathrm{~g}$ ). As the soluble NES probe is cleaved more efficiently than cytoplasmic ones with no access to the plasma membrane, it is very likely cleaved by caspase- 8 at the plasma membrane. These observations were confirmed in HeLa with no CD95 overexpression, MCF-10a, T98G, MDAMB-231 and HT-1080 (Supplementary Figure 3).

In contrast to CD95, TNFR1 was proposed to induce apoptosis through the recruitment and activation of caspase-8 on endosomes, ${ }^{30,31}$ and alternatively through BID cleavage by cathepsins $B$ and $D$ released from the lysosomes into the cytosol. $^{30-34}$ The first mechanism would favor NES probe cleavage over the other localization probes, including the plasma membrane one, while the second mechanism would induce an equal cleavage of all probes in the cytosol, as observed with caspase-3 (Supplementary Figure 2e). HeLa cells expressing localization probes and treated with $100 \mathrm{ng} \mathrm{ml}^{-1}$ TNF $\alpha$ and $5 \mu \mathrm{g} \mathrm{ml}^{-1}$ cycloheximide showed apoptotic bodies after $200+/-100 \mathrm{~min}$. While NES-DEVDR was abruptly cleaved just before cell death, NES-ELQTDG was gradually cleaved, with a late onset at around $150 \mathrm{~min}$ corresponding to $75 \%$ of the time required for apoptotic body formation (Figure 4a). Strikingly, plasma membrane and ER ELQTDG probes were significantly less efficiently cleaved than NES-ELQTDG (Figures $4 b$ and c), suggesting the activity from a non-soluble cytoplasmic enzyme, consistent with the model of caspase-8 activation on endosomes. Taken together, these results clearly show that until apoptotic body formation, caspase- 8 activity is mostly concentrated at the plasma membrane upon CD95 activation and that it can mostly cleave plasma membrane and soluble cytosolic substrates. In contrast, TNF $\alpha$ seems to generate caspase- 8 activity inside the cytoplasm.

Caspase- 6 does not significantly contribute to caspase8 activity before apoptotic body formation. The effector caspase- 6 can cleave caspase- $8 .{ }^{35}$ Although caspase- 6 is expected to be cleaved by caspase- $3,{ }^{36-38}$ it may feedback on caspase- 8 before MOMP as XIAP does not inhibit it, ${ }^{39}$ and as it can activate itself. ${ }^{40,41}$ To investigate if caspase- 6 contributes to caspase- 8 activity upon CD95 activation, we compared caspase-8 probe cleavage with and without it. Caspase- 6 was not detectable in our HeLa cell lines but could be exogenously expressed and cleaved upon CD95 activation (Figures $5 \mathrm{a}$ and b). Apoptotic kinetics was compared by mixing cells overexpressing caspase- 6 with NES-DEVDR-EYFP and control cells expressing NESDEVDR-mCherry. While in HeLa cells, no difference was observed upon CD95 activation (Figure 5c), in HeLa-CD95, caspase- 6 overexpression leads to a slightly faster cell death (Figure 5d), with a shift of 4 min compared with control. Moreover, when caspase- 6 was overexpressed, in HeLa and HeLa-CD95, no significant difference could be observed on the cleavage of NES-, Myr-Palm-snap3- and calnexinELQTDG and NES-DEVDR (Figures $5 e$ and $f$ and Supplementary Figures $4 \mathrm{a}-\mathrm{d}$ ). Therefore, caspase- 6 has no effect on caspase-8 before MOMP. Moreover, by western blot, no effect could be observed on the cleavage of BID and PARP at the cell population level (Supplementary Figures $4 \mathrm{e}$ and f). Caspase- 8 is already significantly cleaved when apoptotic bodies form (Supplementary Figures $4 e$ and f) so the feedback effect may be limited, as already concluded from mathematical modeling of the pathway. ${ }^{42}$ Thus, caspase-6 can slightly accelerate CD95-induced apoptosis but does not affect the early phase of caspase-8 activity.

Caspase- 8 accumulates in plasma membrane and cytoplasmic dotty structures. Caspase-8 probes are mostly cleaved at the plasma membrane, and inefficiently inside the cytoplasm, including on the surface of mitochondria. However, fully processed caspase- 8 was proposed to translocate to the surface of the mitochondria, where it would cleave BID. ${ }^{20,21}$ To test this contradiction, we observed caspase-8-mGFP after 
a

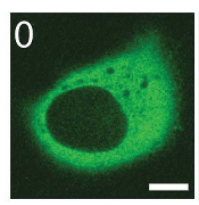

NES-GIETDS-YFP (caspase-3)
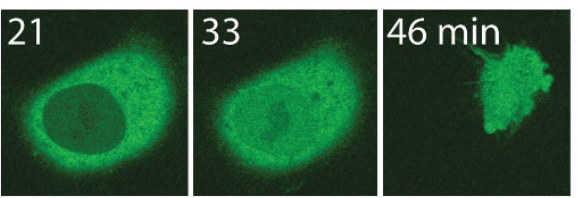

b

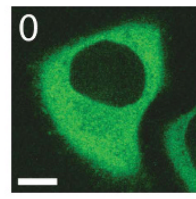

NES-LLEVDG-YFP (cFLIP-L)
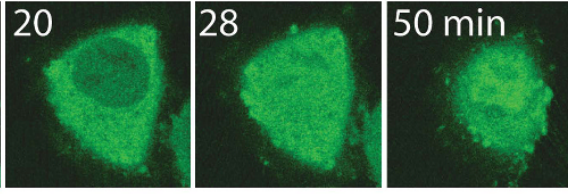

C

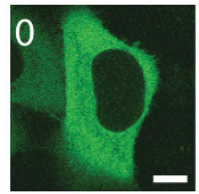

NES-ELQTDG-YFP (BID)
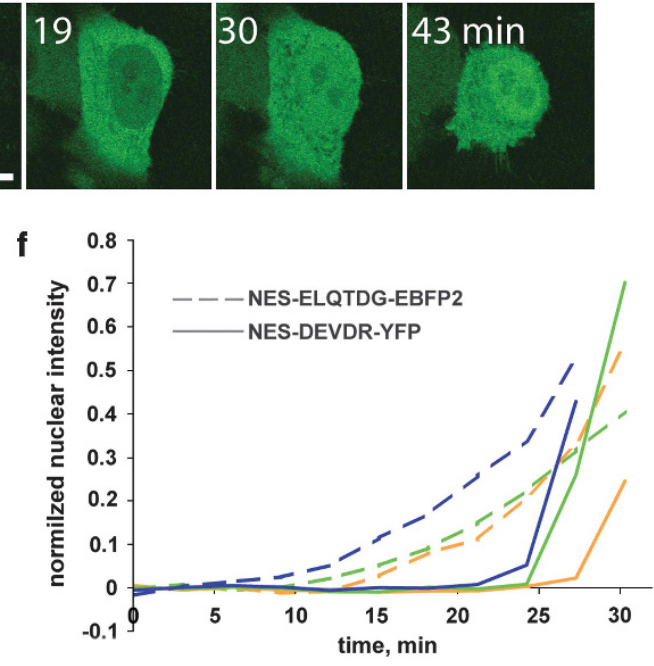

h

NES-ELQTDG-YFP

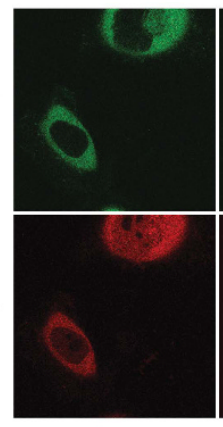

0

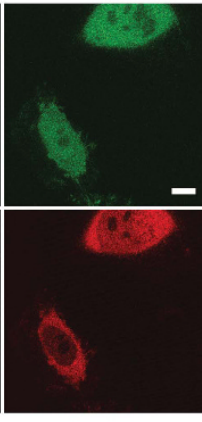

$70 \mathrm{~min}$

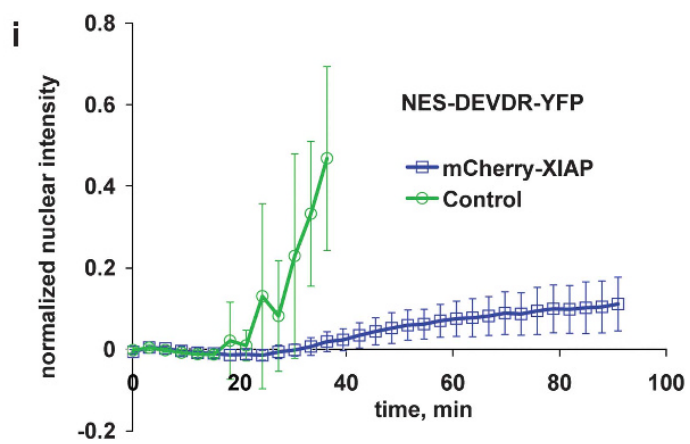

d

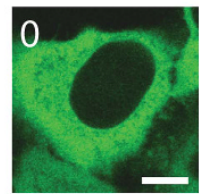

NES-PVETDS-YFP (caspase-8)
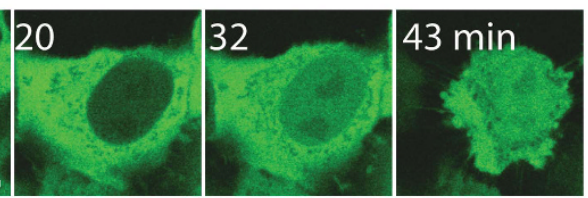

e

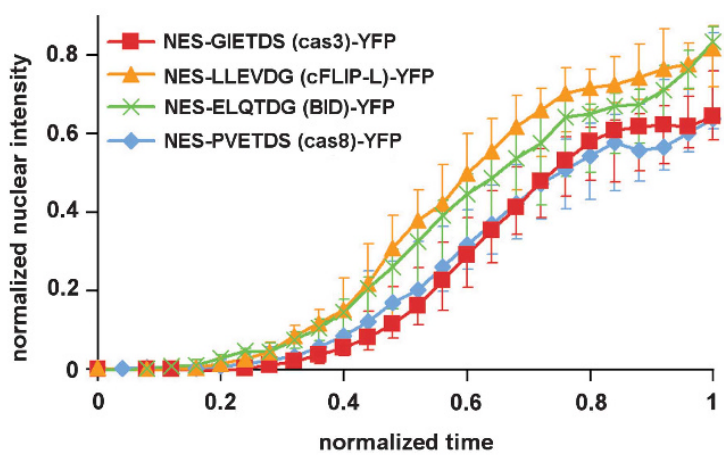

g
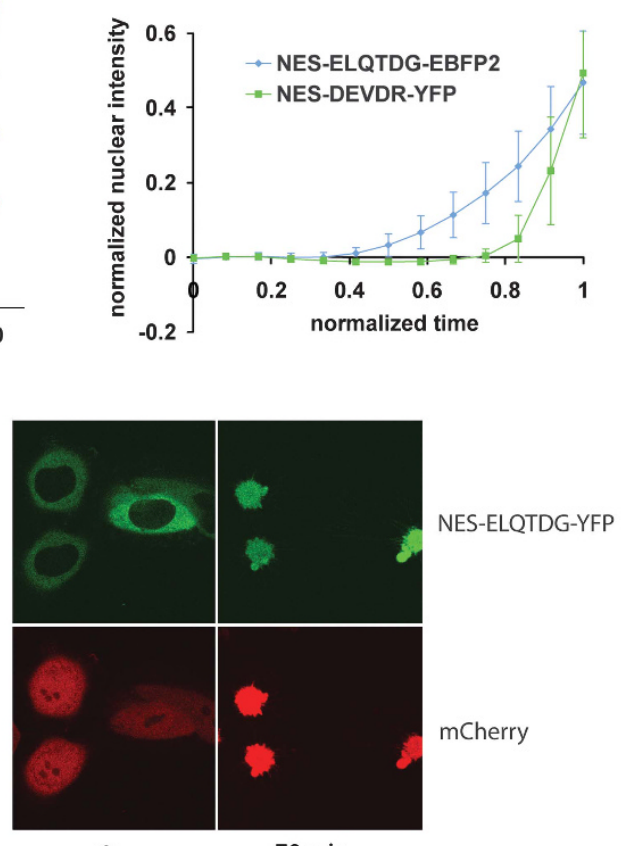

0

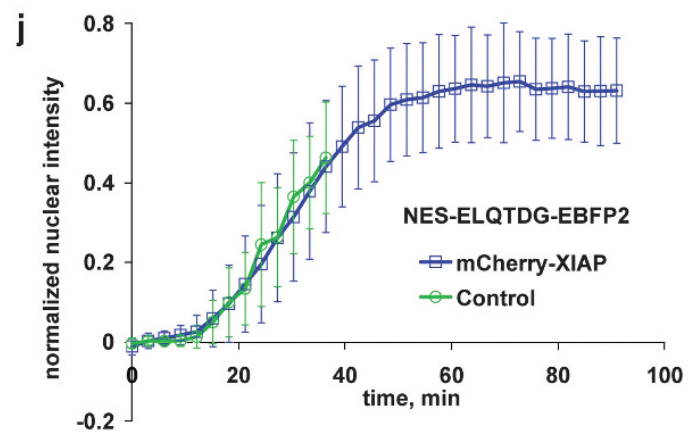


CD95 activation in different cell lines. The functionality of the fusion was confirmed by the restoration of apoptosis sensitivity in HeLa cells, where endogenous caspase-8 was stably knocked-down and caspase-8-mGFP was expressed (Supplementary Figures $5 \mathrm{a}-\mathrm{c}$ ). In this cell line (HeLacas8GFP), the dim and diffuse pattern of caspase-8-mGFP progressively changed to a bright dotty pattern after induction (Figure 6a). Most of the dots could be seen at the periphery of the cells, some of them also appearing inside the cell shortly before apoptotic body formation, potentially correlating with an activity concentrated at the plasma membrane. This observation cannot be generalized as in the neuroblastoma cell line NB7, which lacks endogenous caspase-8, dots of stably expressed caspase-8-mGFP were mostly seen inside the cell after induction (Figure 6b). As apoptotic body formation occurred with little detectable probe cleavage (Supplementary Figure 5d), we could not correlate this observation with activity localization.

Caspase-8-mGFP pattern does not coincide with mitochondria, visualized by Mitotracker-red (Supplementary Figure 5e). In particular, despite the density of the organelle, we could easily find dots outside of the mitochondrial network in HeLa-cas8GFP (Supplementary Figure 5e, white arrows). Moreover, caspase-8-mGFP transiently expressed at very low level in MCF-7 cells also gave a dotty pattern (Supplementary Figure 5f). As this cell line lacks caspase-3, no signs of apoptosis-like membrane blebbing or cell rounding were observed, despite a strong caspase-8 activity visualized by NES-IETD-mCherry cleavage (Supplementary Figure $5 \mathrm{~h}$ ). In addition to the dots, we also observed a more diffuse pattern at later time points, which resembles mitochondria in the caspase-8-mGFP images (Supplementary Figure 5f). But this pattern was also observed in non-transfected cells (Supplementary Figure $5 \mathrm{~g}$ ), clearly indicating that this signal is actually autofluorescence that appears in the same, low range of intensity as caspase-8-mGFP. Taken together, these data show that caspase-8-mGFP is not significantly recruited to mitochondria upon CD95 induction, but accumulates in plasma membrane and cytosolic structures, in a balance that depends on the cell type. Plasma membrane accumulations can be explained by the fact that caspase- 8 accumulates on one FADD at the DISC. ${ }^{43,44}$ Cytosolic accumulations may correspond to endocytosed-activated receptors ${ }^{45}$ or to aggregates driven by ubiquitination, as suggested by the study of Jin et al. ${ }^{46}$

Cytoplasmic caspase-8 activity correlates with caspase8 prodomain cleavage. The limited cytoplasmic activity of caspase-8 observed in several cell lines may correlate with the distribution of caspase- 8 accumulations on the plasma membrane and in the cytoplasm, or with the release of the catalytic domain of caspase-8 from its prodomain, independently of the distribution. To test this hypothesis, we compared the spatio-temporal activity of wild-type caspase8 with a D210A-D216A-D223A mutant that cannot be cleaved between the prodomain and the catalytic domain. We stably expressed the mutants in HeLa cells stably knocked-down for endogenous caspase-8 and kept two stable clones (HeLa-cas8GFP-D210/216/223A-1 and -2). HeLa-cas8GFP, HeLa-cas8GFP-D210/216/223A-1 and -2 showed a similar sensitivity to CD95-induced apoptosis, as already observed in other studies. ${ }^{14}$ Calnexin-ELQTDGmCherry cleavage was too small before apoptotic body formation to allow a direct comparison between the cell lines (Figures $6 \mathrm{c}$ and $\mathrm{d}$ ), therefore, we performed this comparison in cells expressing EBFP2-XIAP to block downstream caspase activity. Both Myr-snap3-ELQTDG-mCherry, normalized to 1 when fully cleaved, and NES-ELQTDGmCherry, normalized to the cytosolic signal at time 0 , showed a strong cleavage in the three clones (Figures $6 \mathrm{e}$ and $\mathrm{f}$ ). In contrast, the amount of calnexin-ELQTDGmCherry, normalized to total intensity at time 0 , was strongly reduced in the two clones of HeLa-cas8GFP-D210/216/223A compared with HeLa-cas8GFP (Figure 6g). This clearly indicates that cytoplasmic caspase- 8 activity is driven by the cleavage of the prodomain of the enzyme.

Targeting of caspase- 8 substrates to the plasma membrane accelerates apoptosis. With caspase- 8 being mostly active at the plasma membrane, the subcellular distribution of its substrates may greatly influence its cleavage efficiency. To test this, we overexpressed BID and caspase-3 at different cellular locations and measured their effect.

MOMP is driven by a threshold mechanism related to the amount of cleaved BID. ${ }^{47}$ Therefore, we expected MOMP and cell death to occur faster with BID overexpression, unless it was targeted to a compartment where it is not efficiently cleaved. To test this, we expressed BID fused to mGFP targeted either to the plasma membrane or to the ER surface and measured the time of apoptotic body formation (Figure 7a). Remarkably, the plasma membrane BID-GFP accelerated cell death compared with the control, while the ER BID-GFP had no effect.

In type II cells, cleaved caspase-3 is normally trapped and kept inactive by XIAP before MOMP. Therefore, one would expect an accelerated apoptosis by caspase-3 overexpression if the cleaved amount of caspase-3 exceeds the amount of XIAP or if caspase- 3 cleavage is so efficient that trapping by $\mathrm{XIAP}$ is not sufficient to prevent caspase-3 activity. To test the

Figure 2 Comparison of specificity for caspase-8 substrates. Cleavage of NES probes linked to EYFP over time, with the cleavage sequences GIETDS for caspase-3 (a), LLEVDG for cFLIP-L (b), LQTDG for BID (c) and PVETDS for caspase-8 (d). The last frame of each sequence corresponds to apoptotic body formation (e). Plots representing the quantification of cleavage of the four probes, as described in Supplementary Material. One can observe that all probes are cleaved with relatively similar rates, starting at the same time and roughly reaching saturation at the time of apoptotic body formation (f). Three examples of cleavage comparison of NES-LQTDG-EBFP2 (dashed line) and NESDEVDR-YFP (solid line) on real-time scale. Each color corresponds to a co-expressed pair of probe within one cell. (g). Same as (f), average on 15 cells, and scaled at 1 for apoptotic body formation (h). Comparison of apoptosis sensitivity with (left) and without (right) mCherry-XIAP. At time 70 min, cells expressing mCherry-XIAP are alive despite full cleavage of NES-LQTDG-YFP (green), while control cells expressing mCherry are dead (i). Comparison of NES-DEVDR-YFP with (blue square) and without (green circle) mCherry-XIAP. The averaging of control cells was stopped at $40 \mathrm{~min}$, when half of the measured cells were apoptotic (j). Comparison of NES-ELQTDG-EBFP2 with (blue square) and without (green circle) mCherry-XIAP as in (i). Note the overlap of the curves until the time, when half of the control cells were apoptotic. Scale bar: $10 \mu \mathrm{m}$ 
a

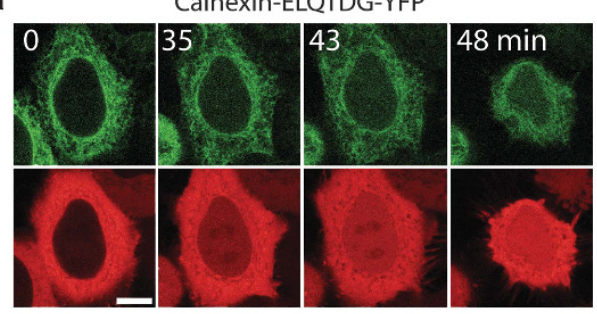

NES-ELQTDG-mCherry

c

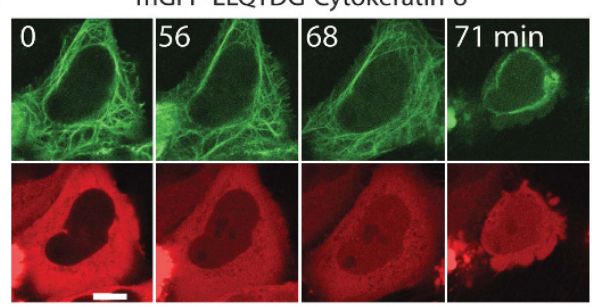

NES-ELQTCG -mCherry b

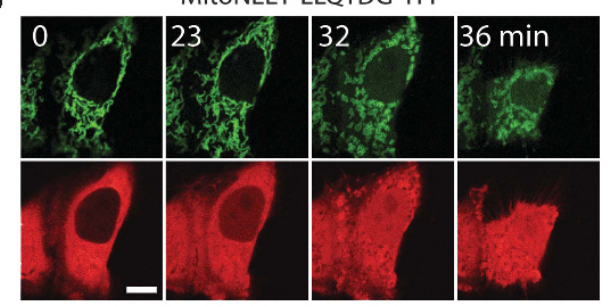

NES-ELQTDG-mCherry

d

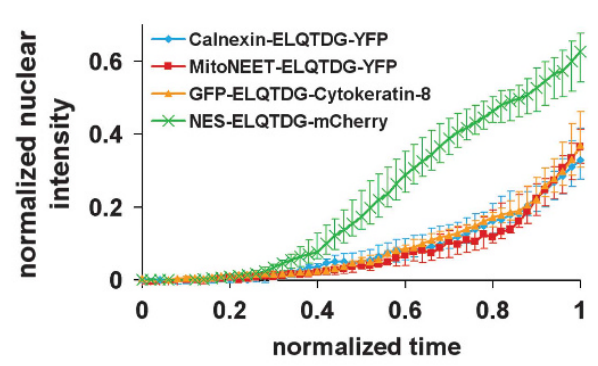

\section{e}

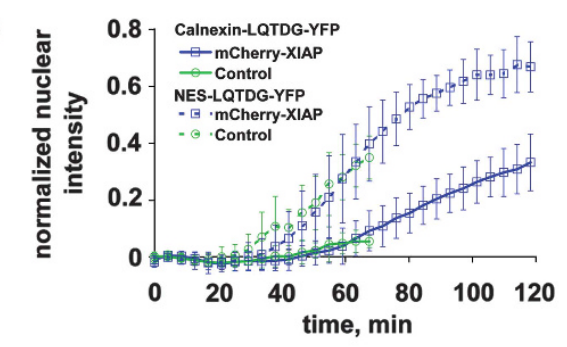

\section{f}

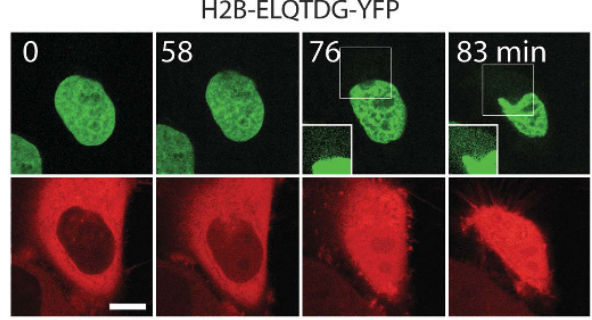

NES-ELQTDG-mCherry
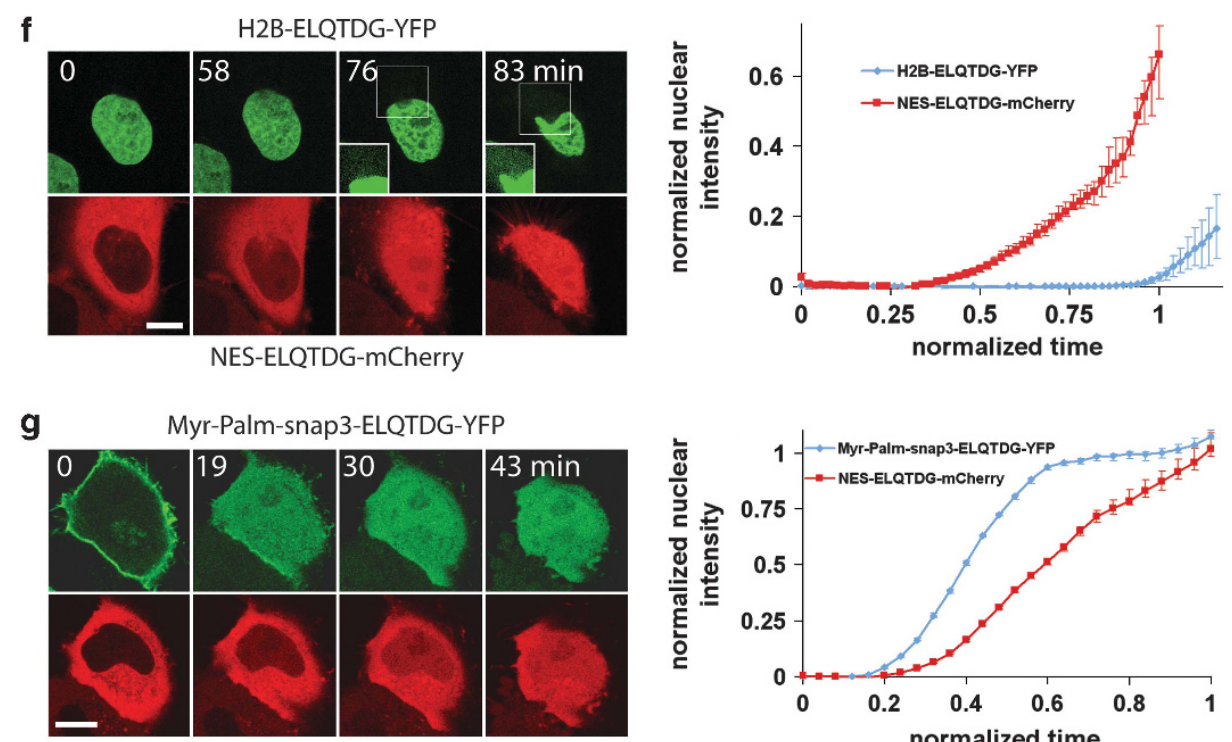

NES-ELQTDG-mCherry

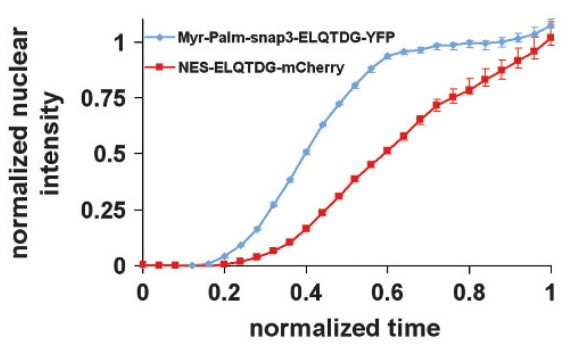

Figure 3 Calnexin (a), MitoNEET (b) and cytokeratin-8 (c) linked to EYFP and mGFP by the sequence LQTDG were compared with NES probes fused to $m$ Cherry by the same linker after CD95 induction (d). Plots of normalized cleavage, as in Figure $2 \mathrm{e}$ for probes shown in a-c. The three plots corresponding to cytoplasmic probes with no access to plasma membrane appeared similar and demonstrate that these probes are much less efficiently cleaved than the NES probe. (e) Comparison of cleavage of NES (dashed lines) and calnexin (solid lines) probe using the LQTDG sequence with (blue squares) and without (green circles) XIAP overexpression. Note that calnexin-LQTDG follows the same kinetics as in control cells until apoptosis of half of the cells. (f) H2B probe cleavage (blue diamond) compared with NES probe cleavage (red squares) with LQTDG cleavage sequence. (g) Same as (f) with the plasma membrane Myr-Palm-snap3 probe (blue diamond). Scale bar: $10 \mu \mathrm{m}$ 
a

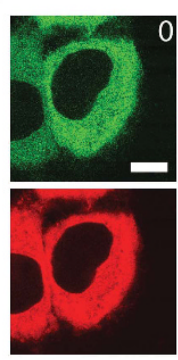

NES-DEVDR-YFP
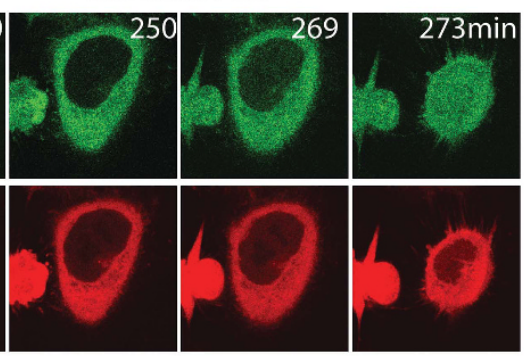

NES-LQTDG-mCherry

b

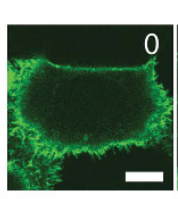

Myr-Palm-snap3-LQTDG-YFP
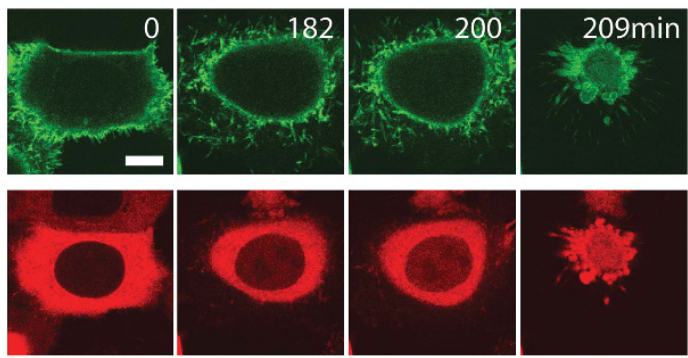

NES-LQTDG-mCherry

C

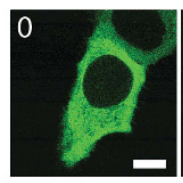

NES-LQTDG-YFP
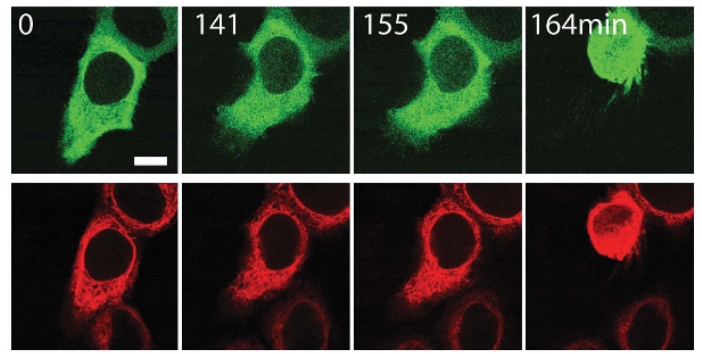

Calnexin-LQTDG-mCherry
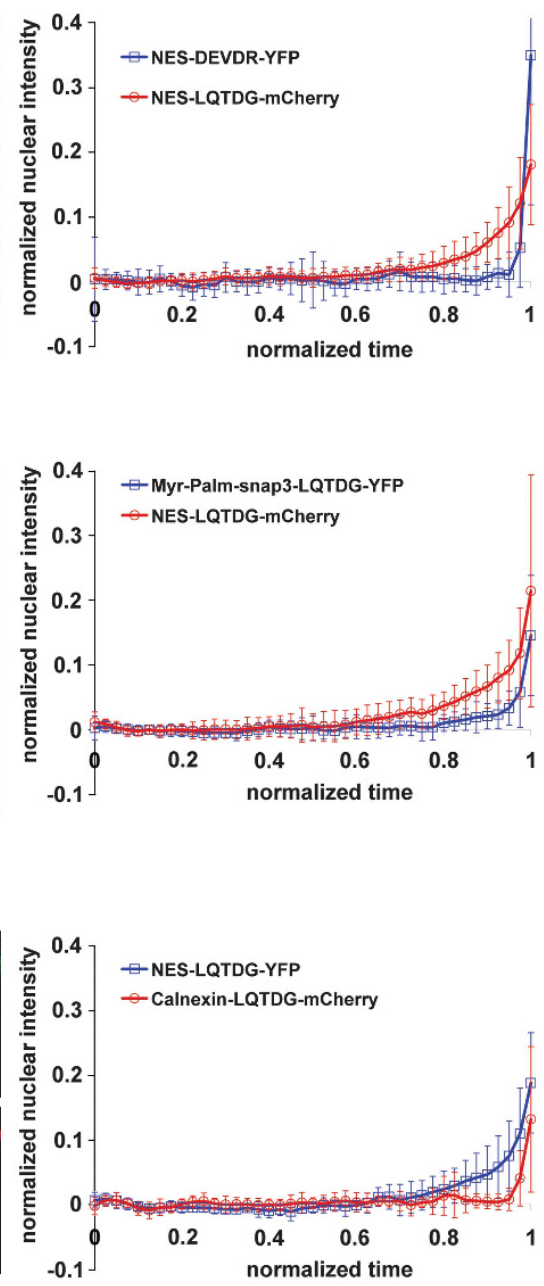

Figure 4 TNF $\alpha+$ cycloheximide induces activity inside the cytosol. (a) Cleavage comparison of NES-DEVDR-YFP and NES-LQTDG-mCherry, as in Figure 2 with TNF $\alpha+$ cycloheximide induction in HeLa cells. (b) Same as (a) with Myr-Palm-snap3-LQTDG-YFP and NES-LQTDG-mCherry. (c) Same as (a) with NES-LQTDG-YFP and calnexin-LQTDG-mCherry. Scale bar: $10 \mu \mathrm{m}$

effect of caspase-3 localization, we transiently transfected HeLa-CD95 with equal amounts of plasmids coding for caspase-3 fused to NES, Myr-Palm or calnexin, or for mGFP as control and measured the time of apoptotic body formation (Figure 7b). While calnexin-caspase-3 did not show any effect compared with control, NES-caspase-3 overexpression slightly accelerated cell death. Strikingly, Myr-Palm-caspase-3 overexpression largely accelerated the process: apoptotic bodies appeared after $15 \mathrm{~min}$ instead of $30 \mathrm{~min}$ in control cells.

To further characterize the mechanisms of transduction of caspase activity that leads to accelerated apoptosis, we compared caspase- 8 and caspase- 3 activity, depending on the targeting of overexpressed caspase-3. HeLa-CD95 were co-transfected with NES-ELQTDG-mCherry and NESDEVDR-GFP, to visualize caspase-8 and -3 activity, respectively, and caspase-3 targeted to the cytoplasm or the plasma membrane (Figures 7c-e). In all cases, cleavage of the ELQTDG probe was similar. Control cells with no caspase-3 overexpression showed a late and abrupt caspase-3 activity (Figure 7c, see also Figures $2 \mathrm{f}$ and g). In contrast, cytosolic caspase-3 overexpression leads to a gradual and early caspase-3 activity (Figure 7d), while plasma membrane caspase-3 overexpression leads to a complete change of activity: caspase- 3 becomes active as soon as caspase-8 activity appears and its activity is immediately stronger than the one of caspase-8, leading to rapid cell death (Figure $7 \mathrm{e}$ ).

Taken together, these results show that both BID and caspase- 3 can be cleaved as full-length proteins preferentially at the plasma membrane, like observed for their corresponding cleavage probes. Our experiments also show that the subcellular concentration of capase- 8 substrates can strongly influence the cellular sensitivity to apoptosis.

\section{Discussion}

In this study, we developed fluorescent probes to spatially and temporally quantify and compare caspase activities in living cells. Existing caspase sensors only partially cover these 


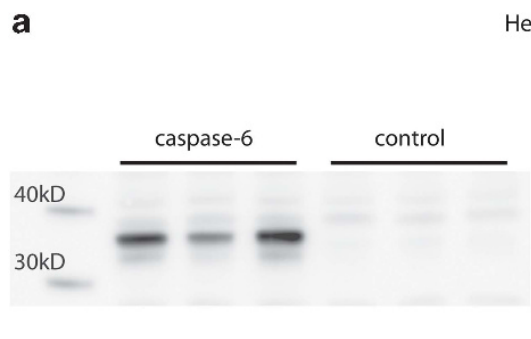

HeLa-CD95

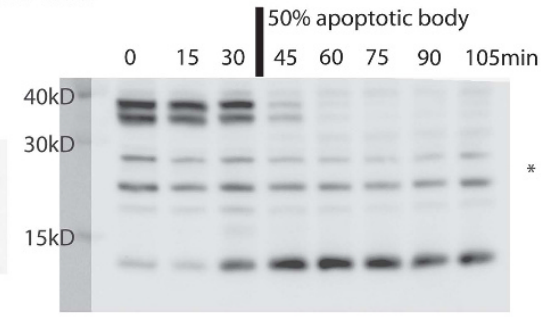

b

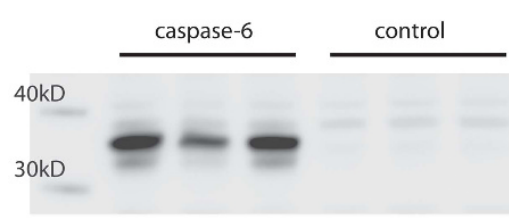

HeLa

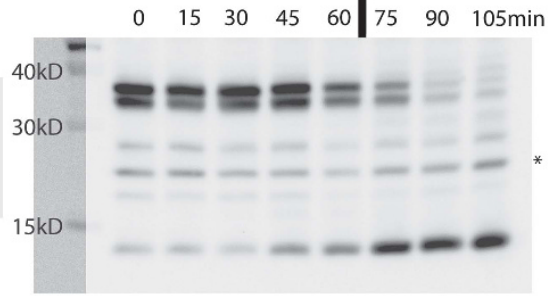

C

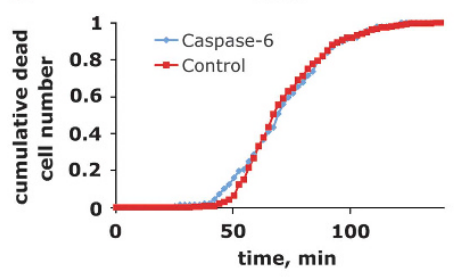

e

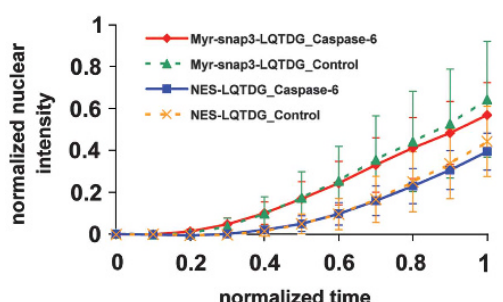

d

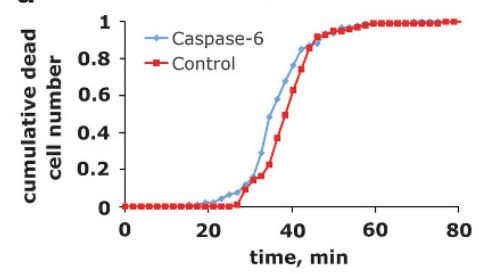

f

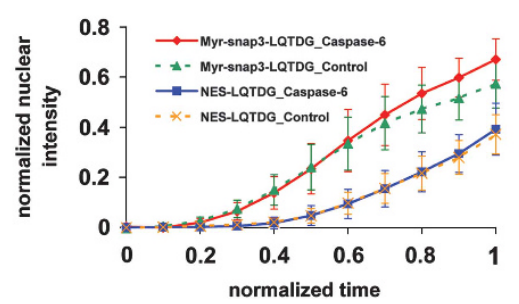

Figure 5 Caspase-6 overexpression does not affect early caspase-8 activity. (a) Comparison by western blotting of caspase-6 expression (expected size: $35 \mathrm{kD}$ ) in HeLaCD95 overexpressing caspase-6 (three left bands) and control cells (three right bands). The three lanes correspond to the three experiments performed with probes, shown on (f) and Supplementary Figures $4 \mathrm{~b}$ and $\mathrm{d}$. The timeseries (right) shows caspase- 6 cleavage (expected size: $15 \mathrm{kD}$ ) over time. The vertical line visualizes the time when around $50 \%$ of the cells were apoptotic as seen by transmission microscopy before lysing the cells. The contrast of the ladder was enhanced on half of its width for better visibility. (b) Same as (a) in HeLa cells. The three lanes correspond to the three experiments performed with probes, shown on (e) and Supplementary Figures 4a and c. (c) Comparison of time of apoptotic body formation measured by microscopy in HeLa cells overexpressing caspase-6 (blue diamond) and control HeLa cells (red square). Note the overlap between the curves. (d) Same as (c) with HeLa-CD95. Note the 4-min shift of time of death when caspase-6 is expressed. Measurement was performed in the same chamber, excluding difference in ligand treatment. (e) Cleavage comparison of Myr-snap3-LQTDG (red diamond and green triangle) and NES-LQTDG (blue square and orange cross) with (solid line) and without (dashed line) caspase-6 overexpression in HeLa cells as in Figure 2. (f) Same as (e) in HeLa-CD95. Scale bar: $10 \mu \mathrm{m}$

needs. Fluorescent inhibitors partially block caspase activity and therefore influence quantification. Moreover, they mostly mark caspase- 8 accumulations ${ }^{19}$ and visualization of diffuse active caspase- 8 requires washing of non-bound sensors. FRET probes, ${ }^{28,48,49}$ which lose their FRET signal after cleavage, allow the measurement of caspase activity in living cells. But measuring different probes at once is difficult because of the spectral requirements, making the comparison between sensors more challenging. The sensors presented here allow this in a straightforward manner within each individual cell: one can compare the efficiency of cleavage of different sequences at different subcellular locations with a sensitivity that is only limited by the sensitivity of the imaging microscope.

Here, the probes were developed to characterize the spatial propagation of caspase-8 activity upon CD95-induction. BID and caspase- 3 cleavage are generally thought to require caspase- 8 release from the DISC. ${ }^{18}$ However, this release induces a loss of activity in vitro. ${ }^{12}$ Moreover, some studies in living cells proposed that caspase- 8 translocates to the mitochondria, ${ }^{20,21}$ another study proposed that it translocates to p62-dependant aggregates ${ }^{46}$ and finally receptor internalization was shown to be required in type I cells. ${ }^{45}$ These three processes would result in cytoplasmic activity. Here, we 
a

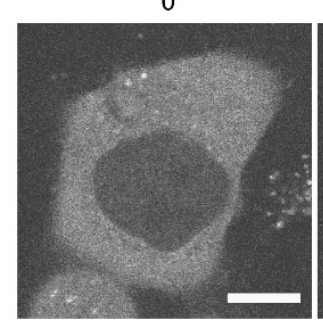

b

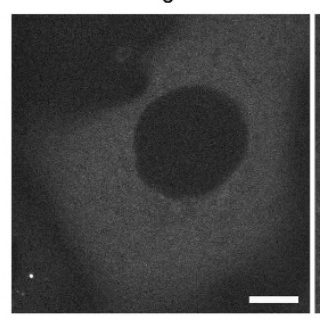

13

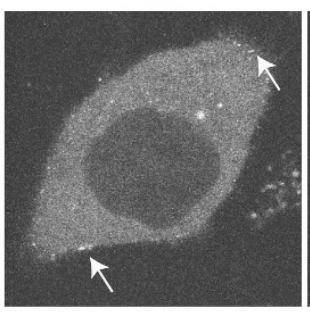

caspase-8-mGFP, HeLa

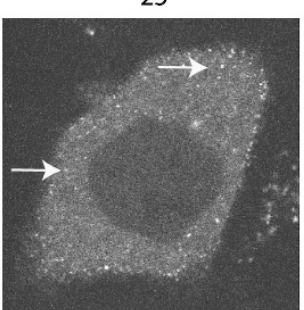

123
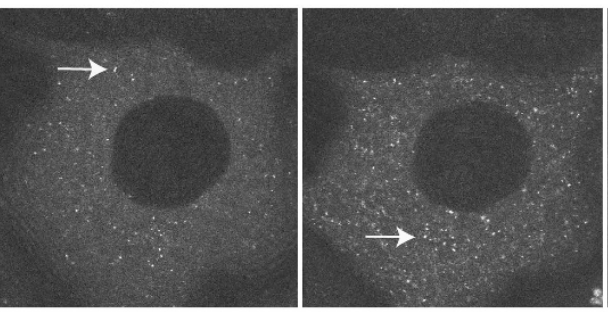

caspase-8-mGFP, NB7

C

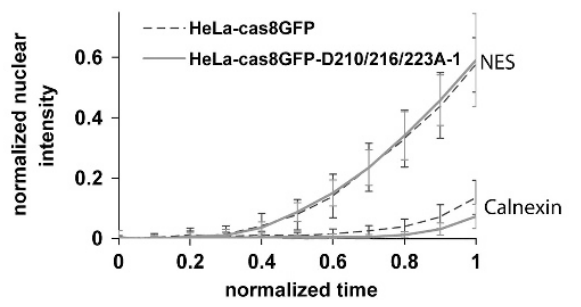

d

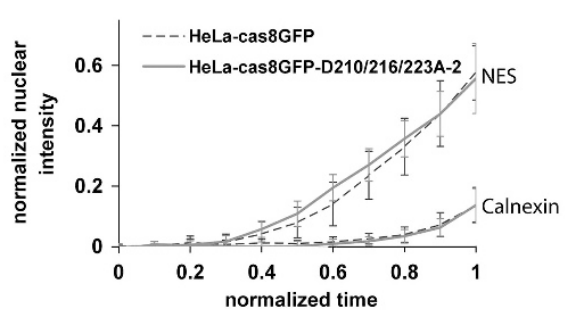

e $\quad 1.2$ Myr-Palm-snap3-LQTDG-mCherry
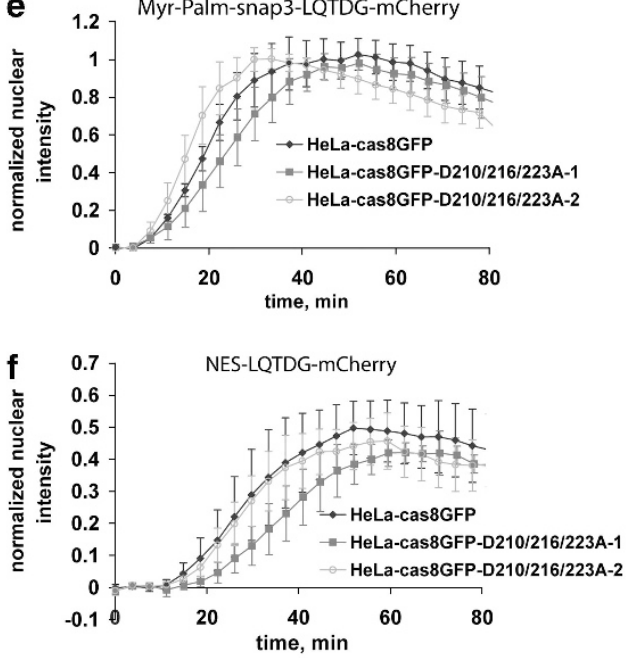

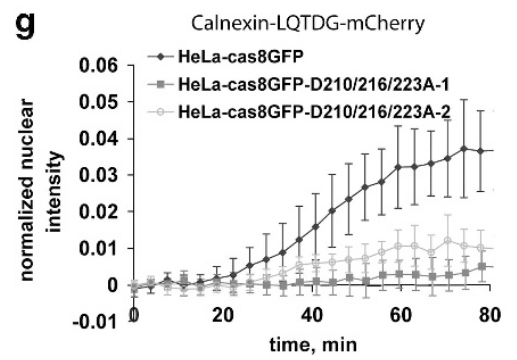

Figure 6 Caspase-8 mGFP forms a plasma membrane and cytosolic dotty pattern after induction. (a) HeLa cells knocked-down for caspase-8 and expressing caspase-8mGFP, monitored after induction. Note the dotty pattern, first exclusively at the periphery (white arrows at $13 \mathrm{~min}$ ) then also inside the cell (white arrows at $25 \mathrm{~min}$ ). (b) NB7 stably expressing caspase-8-mGFP monitored until apoptotic body formation after induction. A dotty pattern can be observed exclusively inside the cell (e.g., white arrow at $123 \mathrm{~min}$ ). (c) Cleavage comparison of NES-LQTDG (upper curves) and calnexin-LQTDG (lower curves) in HeLa-cas8GFP and HeLa-cas8GFP-D210/216/223A-1 as in Figure 2e. (d) Same with HeLa-cas8GFP and HeLa-cas8GFP-D210/216/223A-2. (e) Cleavage comparison of Myr-snap3-LQTDG-mCherry in HeLa-cas8GFP (blue diamond), HeLa-cas8GFP-D210/216/223A-1 (purple square) and HeLa-cas8GFP-D210/216/223A-2 (orange circle) expressing EBFP2-XIAP. Normalization was made to 1, when all probes were cleaved. (f) Same as (e) with NES-LQTDG-mCherry, with normalization with cytosolic intensity at time 0. (g) Same as (e) with calnexin-LQTDG-mCherry, with a normalization with the total intensity at time 0 measured from 3D stacks. Note the reduced amount of calnexin probe cleavage with caspase-8 mutants that cannot be cleaved between their prodomain and catalytic domain. Scale bar: $10 \mu \mathrm{m}$. A full color version of this figure is available at Cell Death and Differentiation journal online 
a

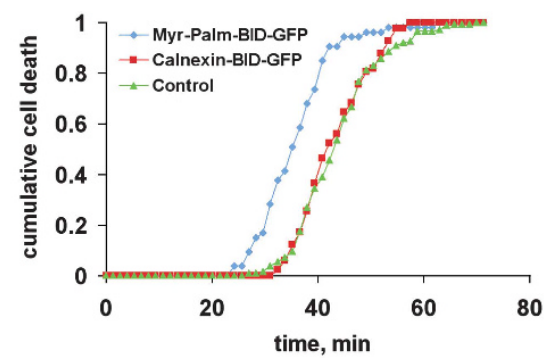

b

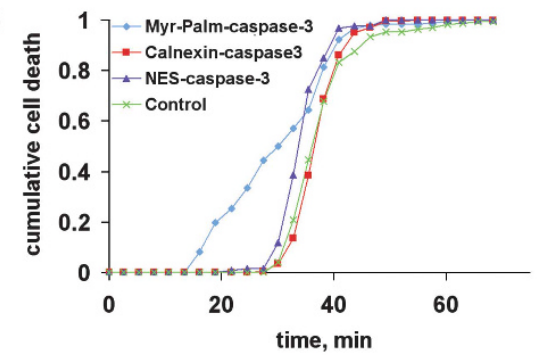

C NES-DEVDR-YFP < casp 3>

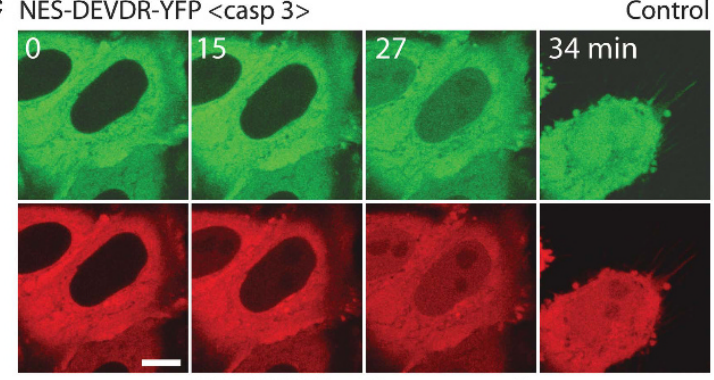

Control

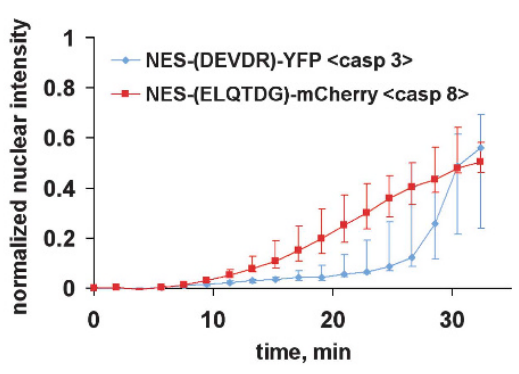

NES-ELQTDG-mCherry $<$ casp 8>

d NES-DEVDR-YFP <casp 3>
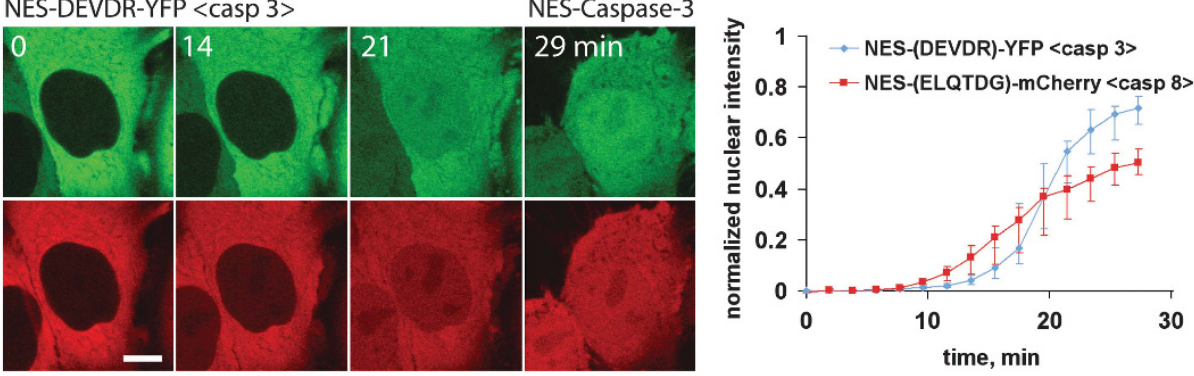

NES-ELQTDG-mCherry <casp 8>

e NES-DEVDR-YFP $<$ casp 3>
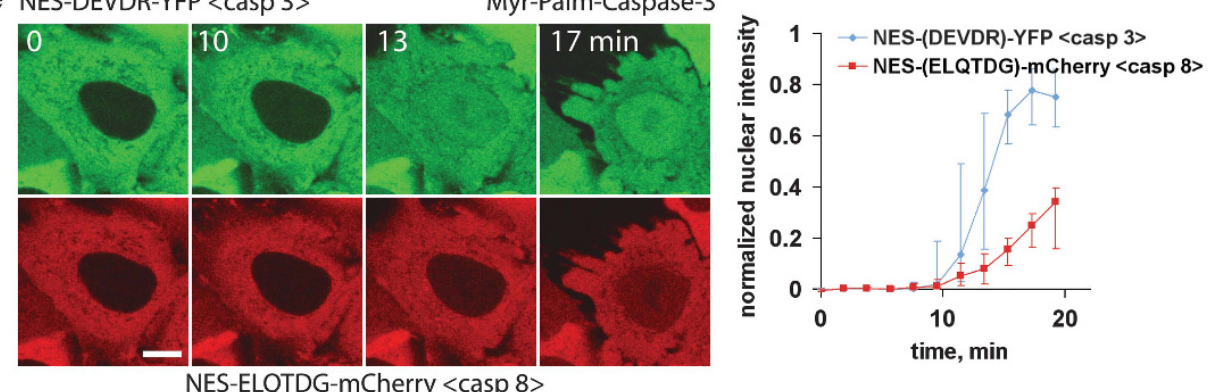

Figure 7 BID and caspase-3 targeted to the plasma membrane, and not to ER, accelerate cell death. (a) Cumulative distribution of time of apoptotic body formation for HeLa-CD95 cells overexpressing BID-GFP targeted to the plasma membrane (Myr-Palm, blue diamond) and ER (calnexin, red square), compared with non-transfected cells as control (green triangle). (b) Same as (a) with caspase-3 as substrate for cleavage. In both (a) and (b), plasma membrane targeting (Myr-Palm, diamond) accelerates cell death. (c-e). Comparison of cleavage of NES-DEVDR-YFP reflecting enzymatic activity of caspase-3 and NES-LQTDG-mCherry reflecting enzymatic activity of caspase-8 coexpressed alone (c) or with NES-caspase-3 (d) or Myr-Palm-caspase-3 (e). Note the stepwise shift of enzymatic activity of caspase-3 (blue diamond) in comparison to enzymatic activity of caspase-8 (red square), when caspase-3 is targeted to the cytosol (d) and the plasma membrane (e) compared with the control (c). Plots of probe cleavage are as described in Figure 2e, however, not applying time normalization. Scale bar: $10 \mu \mathrm{m}$

observed a much more efficient cleavage of probes that can access the plasma membrane compared with the ones that cannot. This clearly favors a model where DISC release of caspase- 8 is not required and where internalization of the enzyme is not involved in cleaving its substrates upon CD95 activation.

The regulation of caspase-8 activity is particularly important in type II cells, where the mitochondrial pathway is required for cell death: caspase- 8 activity initiates the signaling cascade but the cellular decision to die or to survive is determined downstream, notably through the amount of cleaved BID. ${ }^{47}$ This model implies that not only caspase- 8 activation, but also its termination need to be well controlled. Moreover, in case of survival, cellular damage induced by caspase- 8 should be kept minimal. Caspase- 8 being active on the plasma 
membrane and losing its activity upon DISC release would address both aspects. The weak cytoplasmic activity observed here can be because of a loss of activity after prodomain cleavage, as seen in vitro, ${ }^{12}$ or because of a limited release. The enhancement of caspase activity by caspase-8 ubiquitination and translocation to p62 aggregates ${ }^{46}$ apparently contradicts such a model and thus requires further investigations in the future. In particular, combining quantification of caspase activity, cleavage efficiency and localization together with mathematical modeling will help to further unravel the mechanisms that regulate caspase-8 activity and termination after induction.

\section{Materials and Methods}

Cell culture. HeLa-cas8KD was selected with G418 (Sigma-Aldrich, Taufkirchen, Germany). HeLa-CD95 and NB7 stably expressing caspase-8-mGFP were selected with puromycin (Sigma-Aldrich). NB7 were a kind gift of Jill Lahti, St Jude Children's Hospital, Memphis. HeLa, MCF-7, MDA-MB-231, HT-1080, T98G and NB7 were maintained in Dulbecco modified eagle medium (DMEM, Invitrogen, Darmstadt, Germany) containing 10\% fetal calf serum (Biochrom AG, Berlin, Germany), penicillin/streptomycin, $100 \mu \mathrm{g} \mathrm{ml}^{-1}$ each (Invitrogen). MCF-10A was maintained in DMEM with F12 (Invitrogen), $5 \%$ horse serum, $20 \mathrm{ng} \mathrm{ml}^{-1}$ EGF (TEBU-Bio), $0.5 \mu \mathrm{g} \mathrm{ml}^{-1}$ hydrocortisone (Sigma-Aldrich), $10 \mu \mathrm{g} \mathrm{ml}^{-1}$ insulin (Sigma-Aldrich) and penicillin/streptomycin, $100 \mu \mathrm{g} \mathrm{ml}^{-1}$ each (Invitrogen).

For mitochondrial visualization, Mitotracker-red fm (Invitrogen) was preincubated at $50 \mathrm{~nm}$ for $30 \mathrm{~min}$.

Soluble trimerized CD95 ligand was obtained by stably expressing LZ-SCD95L in $293 T$ cells after puromycin selection. Induction of apoptosis was achieved by replacing the medium of the HeLa cells by supernatant of the stable 293T cells maintained without puromycin. TNF $\alpha$ was from Invitrogen and cycloheximide from Sigma-Aldrich. Cells were grown on chambered coverglass (Nunc, Langenselbold, Germany), transfected $24 \mathrm{~h}$ later using Fugene6 (Roche, Basel, Switzerland) and imaged $48 \mathrm{~h}$ after transfection.

DNA constructs. CD95 and caspase- 8 were kindly provided by Peter Krammer, DKFZ Heidelberg. Cytokeratin- $8^{50}$ was a kind gift from Harald Herrmann, DKFZ Heidelberg, MitoNEET and calnexin were kindly provided by Jack Dixon, UCSD San Diego, and Giulia Baldini UAMS Little Rock. H2B-CFP ${ }^{5}$ was a kind gift of Jan Ellenberg, EMBL Heidelberg. mGFP ${ }^{52}$ and mCherry ${ }^{53}$ were kindly provided by Jennifer Lippincott-Schwartz, NIH Bethesda, and Roger Tsien, UCSD San Diego, respectively. BID and caspase-3 were kindly provided by Abelardo Lopez-Rivas ${ }^{54}$ and Martin Sprick, DKFZ Heidelberg.

NES was designed with the MNLVDLQKKLEELELDEQQ sequence. Myr-Palm domain was constructed with the MGCIKSKRKDNLNDDE sequence as described in Zocharias et al. ${ }^{55}$ The aspartic acid at position 81 of cytokeratin-8, which can be cleaved by caspases, was mutated to an alanine using the QuikChange XL sitedirected mutagenesis kit (Stratagene; Agilent Technologies, Waldbronn, Germany).

Cytokeratin-8 was fused to mGFP on its $N$-terminal with the linker GVGGSGGXXXXXGGGGRVGGG, XXXXX being the cleavage sequence mentioned in the text. NES and Myr-Palm were fused on their $\mathrm{C}$-terminal end to fluorescent proteins with the linkers TGGXXXXXGGGGG, H2B with PPGSTG GXXXXXGGGGG, calnexin with GISSSSFEFCSRRYRGPGGXXXXXGGGGG and MitoNEET with VPGGGGSGGXXXXXGGGGG. Myr-Palm-(SNAP)n- probes were made by inserting SNAP tags (New England Biolabs, Frankfurt am Main, Germany) between the Myr-Palm and the cleavage site, with the linkers TGGSG and PPGSTGGXXXXXGGGGG. Clonings were performed in the vectors pEGFP N1 and $C 1$ (Takara Bio Europe Clontech, Saint-Germain-en-Laye, France), where EGFP was replaced by mGFP, EYFP, EBFP2 and mCherry, respectively. For controls with NES and Myr-Palm the linker GGPDEVARGGGGG was used.

For Myr-Palm-BID-GFP and calnexin-BID-GFP, the cleavage linker and the fluorescent protein were replaced by BID fused to MGFP. The same was done for Myr-Palm-, calnexin- and Nes-caspase-3 using the full-length caspase-3.

CD95 was cloned in the pIRES-puro2 vector (Takara Bio Europe Clontech). shRNA targeted against the sequence $5^{\prime}$-GGTCATGCTCTATCAGATT- $3^{\prime}$ of caspase-8 was cloned in pSilencer3.1-neo (Applied Biosystems; Life Technologies $\mathrm{GmbH}$, Darmstadt, Germany). For caspase-8 expression, the sequence normally targeted by the shRNA was mutated to AGTTATGTTGTACCAAATT, which conserved the amino acid sequence. Caspase-8 fusion to mGFP was made with the linker GGGGGIPVAT and was cloned in the pIRES-puro2 vector (Takara Bio Europe Clontech). Caspase-8 and caspase-8-mGFP were also cloned at the place of the neomycin phosphotransferase gene in pIRES-Ne03, where the EBFP2 gene was cloned in the multiple cloning site. XIAP (Addgene, Cambridge, MA, USA) was fused on its $N$-terminal part to EBFP2 and mCherry with the linker SGLRSRARIDICGLASGSAT. Caspase-6 (Addgene) was cloned in pIRES-Neo3. LZ-sCD95L ${ }^{56}$ was cloned in pIRES-puro2.

Microscopy. Experiments were performed on a Leica SP2 laser scanning confocal microscope (LSCM) (Leica Microsystems CMS GmbH, Mannheim, Germany) using a proprietary macro for auto-focusing and multi-position imaging. The temperature was maintained at $37^{\circ} \mathrm{C}$ and media were buffered with $25 \mathrm{~mm}$ HEPES. Alternatively, experiments were performed on a Leica SP5 LSCM using the Leica Live Data Mode wizard. In this case, $\mathrm{CO} 2$ was maintained at $5 \%$. mCherry was excited with a HeNe laser at $594 \mathrm{~nm}$ and detected between 600 and $650 \mathrm{~nm}, \mathrm{mGFP}$ and EYFP were excited with the 488 and $514 \mathrm{~nm}$ lines of an argon laser and detected between 500 and $570 \mathrm{~nm}$, and 520 and $570 \mathrm{~nm}$, respectively. Time-series were acquired in two dimensions with a time step of 1-2 min. They were focused in the middle of the nucleus using an auto-focus on the basis of glass reflection. Pinhole size was set to 1.3 Airy unit to avoid cytoplasmic signal from below and above the nucleus. Cells expressing Myr-Palm probes were often in contact with each other and the quantification of the total intensity of a single cell was not possible in such cases. To do so, neighboring cells were photobleached beforehand.

Western blot. Western blot timeseries were performed by lysing induced cells at different time points using ice-cooled lysis buffer $(20 \mathrm{~mm} T r i s / \mathrm{HCl}, \mathrm{pH} 7.5$, $150 \mathrm{~mm} \mathrm{NaCl}, 1 \mathrm{~mm}$ phenylmethylsulfonyl fluoride (Sigma-Aldrich), protease inhibitor cocktail, 1\% Triton X-100 (Serva, Mannheim, Germany), and 10\% glycerol). Lysates were analyzed using SDS-PAGE gels. The different time points were loaded in a random manner to avoid quantification bias. Proteins were transferred to PVDF membrane (Millipore, Billerica, MA, USA) using wet blotting. The primary antibody against GFP was the mix of clones 7.1 and 13.1 (Roche) and the rabbit anti-BID (\#2002), anti-caspase-6 (\#9762) and anti-PARP (\#9542) antibodies were from Cell Signaling (Danvers, MA, USA). The C15 antibody against caspase-8 was a kind gift of Peter Krammer. Secondary anti-mouse and anti-rabbit antibodies were HRP-conjugated antibodies (Dianova, Hamburg, Germany). Detection was performed using the Pico Chemiluminescent Substrate from Thermo Scientific (Asheville, NC, USA) and a CCD camera.

Acknowledgements. We are grateful to Stefan Kallenberger, Dr Inna Lavrik, Prof Peter Krammer, Dr Nathan Brady, and Dr Stefan Legewie (DKFZ Heidelberg) for critical comments and discussion of this study. This work was supported by the Initiative and Networking Fund of the Helmholtz Association within the Helmholtz Alliance on Systems Biology/ SBCancer. We further acknowledge support by the BMBF funded ForSys centre Viroquant. JB received support by the Center for Modeling and Simulation in the Biosciences (BIOMS).

1. Degterev A, Boyce M, Yuan J. A decade of caspases. Oncogene 2003; 22: 8543-8567.

2. Grutter MG. Caspases: key players in programmed cell death. Curr Opin Struct Biol 2000; 10: 649-655.

3. Siegel RM. Caspases at the crossroads of immune-cell life and death. Nat Rev Immunol 2006; 6: 308-317.

4. Fuentes-Prior $P$, Salvesen GS. The protein structures that shape caspase activity, specificity, activation and inhibition. Biochem J 2004; 384(Pt 2): 201-232.

5. Boatright KM, Renatus M, Scott FL, Sperandio S, Shin H, Pedersen IM et al. A unified model for apical caspase activation. Mol Cell 2003 2003/2 11: 529-541.

6. van Raam BJ, Salvesen GS. Proliferative versus apoptotic functions of caspase-8 Hetero or homo: the caspase-8 dimer controls cell fate. Biochim Biophys Acta 2012; 1824: 113-122.

7. Kischkel FC, Hellbardt S, Behrmann I, Germer M, Pawlita M, Krammer PH et al. Cytotoxicity-dependent APO-1 (Fas/CD95)-associated proteins form a death-inducing signaling complex (DISC) with the receptor. EMBO J 1995; 14: 5579-5588.

8. Boldin MP, Goncharov TM, Goltsev YV, Wallach D. Involvement of MACH, a novel MORT1/FADD-interacting protease, in Fas/APO-1- and TNF receptor-induced cell death. Cell 1996; 85: 803-815. 
9. Muzio M, Chinnaiyan AM, Kischkel FC, O'Rourke K, Shevchenko A, Ni J et al. FLICE, a novel FADD-homologous ICE/CED-3-like protease, is recruited to the CD95 (Fas/APO-1) death-inducing signaling complex. Cell 1996; 85: 817-827.

10. Scaffidi C, Fulda S, Srinivasan A, Friesen C, Li F, Tomaselli KJ et al. Two CD95 (APO-1/ Fas) signaling pathways. EMBO J 1998; 17: 1675-1687.

11. Wachmann K, Pop C, van Raam BJ, Drag M, Mace PD, Snipas SJ et al. Activation and specificity of human caspase-10. Biochemistry 2010; 49: 8307-8315.

12. Hughes MA, Harper N, Butterworth M, Cain K, Cohen GM, MacFarlane M. Reconstitution of the death-inducing signaling complex reveals a substrate switch that determines CD95mediated death or survival. Mol Cell 2009; 35: 265-279.

13. Keller N, Mares J, Zerbe O, Grutter MG. Structural and biochemical studies on procaspase8: new insights on initiator caspase activation. Structure 2009; 17: 438-448.

14. Oberst A, Pop C, Tremblay AG, Blais Vr, Denault J-B, Salvesen GS et al. Inducible dimerization and inducible cleavage reveal a requirement for both processes in caspase-8 activation. J Biol Chem 2010; 285: 16632-16642.

15. Kang TB, Oh GS, Scandella E, Bolinger B, Ludewig B, Kovalenko A et al. Mutation of a selfprocessing site in caspase-8 compromises its apoptotic but not its nonapoptotic functions in bacterial artificial chromosome-transgenic mice. J Immunol 2008; 181: 2522-2532.

16. Keller N, Grutter MG, Zerbe $O$. Studies of the molecular mechanism of caspase-8 activation by solution NMR. Cell Death Differ 2010; 17: 710-718.

17. Golks A, Brenner D, Schmitz I, Watzl C, Krueger A, Krammer PH et al. The role of CAP3 in CD95 signaling: new insights into the mechanism of procaspase-8 activation. Cell Death Differ 2006; 13: 489-498.

18. Martin DA, Siegel RM, Zheng L, Lenardo MJ. Membrane oligomerization and cleavage activates the caspase-8 (FLICE/MACHalpha1) death signal. J Biol Chem 1998; 273: 4345-4349.

19. Koenig A, Russell JQ, Rodgers WA, Budd RC. Spatial differences in active caspase-8 defines its role in T-cell activation versus cell death. Cell Death Differ 2008; 15: 1701-1711.

20. Gonzalvez F, Schug ZT, Houtkooper RH, MacKenzie ED, Brooks DG, Wanders RJ et al. Cardiolipin provides an essential activating platform for caspase-8 on mitochondria. J Cell Biol 2008; 183: 681-696.

21. Schug ZT, Gonzalvez F, Houtkooper RH, Vaz FM, Gottlieb E. BID is cleaved by caspase-8 within a native complex on the mitochondrial membrane. Cell Death Differ 2011; 18 538-548.

22. Kantari $\mathrm{C}$, Walczak H. Caspase-8 and bid: caught in the act between death receptors and mitochondria. Biochim Biophys Acta 2011; 1813: 558-563.

23. Strasser $A$, Jost PJ, Nagata $S$. The many roles of FAS receptor signaling in the immune system. Immunity 2009; 30: 180-192.

24. Granell S, Baldini G, Mohammad S, Nicolin V, Narducci P, Storrie B. Sequestration of mutated alpha1-antitrypsin into inclusion bodies is a cell-protective mechanism to maintain endoplasmic reticulum function. Mol Biol Cell 2008; 19: 572-586.

25. Wiley SE, Murphy AN, Ross SA, van der Geer P, Dixon JE. MitoNEET is an iron-containing outer mitochondrial membrane protein that regulates oxidative capacity. Proc Natl Acad Sci USA 2007; 104: 5318-5323.

26. Chen Y, MacDonald PJ, Skinner JP, Patterson GH, Muller JD. Probing nucleocytoplasmic transport by two-photon activation of PA-GFP. Microsc Res Tech 2006; 69: 220-226.

27. Wei X, Henke VG, Strubing C, Brown EB, Clapham DE. Real-time imaging of nuclear permeation by EGFP in single intact cells. Biophys $J$ 2003; 84(2 Pt 1): 1317-1327.

28. Albeck JG, Burke JM, Aldridge BB, Zhang M, Lauffenburger DA, Sorger PK. Quantitative analysis of pathways controlling extrinsic apoptosis in single cells. Mol Cell 2008; 30: $11-25$.

29. Stennicke HR, Renatus M, Meldal M, Salvesen GS. Internally quenched fluorescent peptide substrates disclose the subsite preferences of human caspases 1, 3, 6, 7 and 8 . Biochem J 2000; 350(Pt 2): 563-568.

30. Micheau O, Tschopp J. Induction of TNF receptor I-mediated apoptosis via two sequential signaling complexes. Cell 2003; 114: 181-190.

31. Tchikov V, Bertsch U, Fritsch J, Edelmann B, Schutze S. Subcellular compartmentalization of TNF receptor-1 and CD95 signaling pathways. Eur J Cell Biol 2011; 90: 467-475.

32. Boya P, Kroemer G. Lysosomal membrane permeabilization in cell death. Oncogene 2008; 27: 6434-6451.

33. Caruso JA, Mathieu PA, Joiakim A, Zhang H, Reiners JJ Jr. Aryl hydrocarbon receptor modulation of tumor necrosis factor-alpha-induced apoptosis and lysosomal disruption in a hepatoma model that is caspase-8-independent. J Biol Chem 2006; 281 : 10954-10967.

34. Heinrich M, Neumeyer J, Jakob M, Hallas C, Tchikov V, Winoto-Morbach S et al. Cathepsin $D$ links TNF-induced acid sphingomyelinase to Bid-mediated caspase- 9 and -3 activation. Cell Death Differ 2004; 11: 550-563.

35. Cowling V, Downward J. Caspase- 6 is the direct activator of caspase- 8 in the cytochrome c-induced apoptosis pathway: absolute requirement for removal of caspase- 6 prodomain. Cell Death Differ 2002; 9: 1046-1056.

36. Inoue S, Browne G, Melino G, Cohen GM. Ordering of caspases in cells undergoing apoptosis by the intrinsic pathway. Cell Death Differ 2009; 16: 1053-1061.

37. Slee EA, Adrain C, Martin SJ. Executioner caspase-3, -6, and -7 perform distinct, nonredundant roles during the demolition phase of apoptosis. J Biol Chem 2001; 276: 7320-7326.

38. Slee EA, Harte MT, Kluck RM, Wolf BB, Casiano CA, Newmeyer DD et al. Ordering the cytochrome c-initiated caspase cascade: hierarchical activation of caspases-2, -3, -6, -7, -8 , and -10 in a caspase-9-dependent manner. J Cell Biol 1999; 144: 281-292.

39. Deveraux QL, Takahashi R, Salvesen GS, Reed JC. X-linked IAP is a direct inhibitor of celldeath proteases. Nature 1997; 388: 300-304.

40. Klaiman G, Champagne N, LeBlanc AC. Self-activation of Caspase- 6 in vitro and in vivo: caspase-6 activation does not induce cell death in HEK293T cells. Biochim Biophys Acta 2009; 1793: 592-601.

41. Wang XJ, Cao Q, Liu X, Wang KT, Mi W, Zhang $Y$ et al. Crystal structures of human caspase 6 reveal a new mechanism for intramolecular cleavage self-activation. EMBO Rep 2010; 11: 841-847.

42. Albeck JG, Burke JM, Spencer SL, Lauffenburger DA, Sorger PK. Modeling a snap-action, variable-delay switch controlling extrinsic cell death. PLOS Biol 2008; 6: 2831-2852.

43. Dickens LS, Boyd RS, Jukes-Jones R, Hughes MA, Robinson GL, Fairall L et al. A death effector domain chain disc model reveals a crucial role for caspase-8 chain assembly in mediating apoptotic cell death. Mol Cell 2012; 47: 291-305.

44. Schleich K, Warnken U, Fricker N, Ozturk S, Richter P, Kammerer K et al. Stoichiometry of the CD95 death-inducing signaling complex: experimental and modeling evidence for a death effector domain chain model. Mol Cell 2012; 47: 306-319.

45. Lee KH, Feig C, Tchikov V, Schickel R, Hallas C, Schutze S et al. The role of receptor internalization in CD95 signaling. EMBO J 2006; 25: 1009-1023.

46. Jin Z, Li Y, Pitti R, Lawrence D, Pham VC, Lill JR et al. Cullin3-based polyubiquitination and p62-dependent aggregation of caspase-8 mediate extrinsic apoptosis signaling. Cell 2009; 137: 721-735.

47. Hellwig CT, Kohler BF, Lehtivarjo AK, Dussmann H, Courtney MJ, Prehn JH et al. Real time analysis of tumor necrosis factor-related apoptosis-inducing ligand/cycloheximideinduced caspase activities during apoptosis initiation. J Biol Chem 2008; 283: 21676-21685.

48. Rehm M, Dussmann H, Janicke RU, Tavare JM, Kogel D, Prehn JHM. Single-cell fluorescence resonance energy transfer analysis demonstrates that caspase activation during apoptosis is a rapid process. J Biol Chem 2002; 277: 24506-24514.

49. Rehm M, Huber HJ, Dussmann H, Prehn JH. Systems analysis of effector caspase activation and its control by X-linked inhibitor of apoptosis protein. EMBO J 2006; 25: 4338-4349.

50. Reichenzeller M, Burzlaff $A$, Lichter $P$, Herrmann $H$. In vivo observation of a nuclear channel-like system: evidence for a distinct interchromosomal domain compartment in interphase cells. J Struct Biol 2000; 129: 175-185.

51. Beaudouin J, Gerlich D, Daigle N, Eils R, Ellenberg J. Nuclear envelope breakdown proceeds by microtubule-induced tearing of the lamina. Cell 2002; 108: 83-96.

52. Snapp EL, Hegde RS, Francolini M, Lombardo F, Colombo S, Pedrazzini E et al. Formation of stacked ER cisternae by low affinity protein interactions. J Cell Biol 2003; 163: 257-269.

53. Shaner NC, Campbell RE, Steinbach PA, Giepmans BN, Palmer AE, Tsien RY. Improved monomeric red, orange and yellow fluorescent proteins derived from Discosoma sp. red fluorescent protein. Nat Biotechnol 2004; 22: 1567-1572.

54. Ortiz-Ferron G, Tait SW, Robledo G, de Vries E, Borst J, Lopez-Rivas A. The mitogenactivated protein kinase pathway can inhibit TRAIL-induced apoptosis by prohibiting association of truncated Bid with mitochondria. Cell Death Differ 2006; 13: 1857-1865.

55. Zacharias DA, Violin JD, Newton AC, Tsien RY. Partitioning of lipid-modified monomeric GFPs into membrane microdomains of live cells. Science 2002; 296: 913-916.

56. Walczak H, Miller RE, Ariail K, Gliniak B, Griffith TS, Kubin M et al. Tumoricidal activity of tumor necrosis factor-related apoptosis-inducing ligand in vivo. Nat Med 1999; 5: 157-163.

Supplementary Information accompanies the paper on Cell Death and Differentiation website (http://www.nature.com/cdd) 